\title{
Ventral Hippocampal Inputs Preferentially Drive Corticocortical Neurons in the Infralimbic Prefrontal Cortex
}

\author{
Xingchen Liu and Adam G. Carter \\ Center for Neural Science, New York University, New York, New York 10003
}

Inputs from the ventral hippocampus (vHPC) to the prefrontal cortex (PFC) play a key role in working memory and emotional control. However, little is known about how excitatory inputs from the vHPC engage different populations of neurons in the PFC. Here we use optogenetics and whole-cell recordings to study the cell-type specificity of synaptic connections in acute slices from the mouse PFC. We first show that vHPC inputs target pyramidal neurons whose cell bodies are located in layer (L)2/3 and L5 of infralimbic (IL) PFC, but only in L5 of prelimbic (PL) PFC, and not L6 of either IL or PL. We then compare connections onto different classes of projection neurons located in these layers and subregions of PFC. We establish vHPC inputs similarly contact corticocortical (CC) and cortico-amygdala neurons in L2/3 of IL, but preferentially target CC neurons over cortico-pontine neurons in L5 of both IL and PL. Of all these neurons, we determine that vHPC inputs are most effective at driving action potential (AP) firing of CC neurons in L5 of IL. We also show this connection exhibits frequency-dependent facilitation, with repetitive activity enhancing AP firing of IL L5 CC neurons, even in the presence of feedforward inhibition. Our findings reveal how vHPC inputs engage defined populations of projection neurons in the PFC, allowing preferentially activation of the intratelencephalic network.

Key words: circuit; hippocampus; interneuron; prefrontal cortex; projection neuron; synapse

Significance Statement

We examined the impact of connections from the ventral hippocampus (vHPC) onto different projection neurons in the mouse prefrontal cortex (PFC). We found vHPC inputs were strongest at corticocortical neurons in layer 5 of infralimbic PFC, where they robustly evoked action potential firing, including during repetitive activity with intact feedforward inhibition.

\section{Introduction}

The prefrontal cortex (PFC) mediates the top-down control of diverse high-level cognitive and emotional behaviors (Euston et al., 2012). Local processing in the PFC is driven by multiple longrange excitatory inputs that arrive from other brain regions (Miller and Cohen, 2001; Hoover and Vertes, 2007). Connections from the ventral hippocampus (vHPC) to the PFC are thought to be particularly important for the regulation of both short-term and long-term memories (Siapas et al., 2005; Fanselow and Dong, 2010; Sotres-Bayon et al., 2012; O’Neill et al., 2013; Spellman et al., 2015; Kim and Cho, 2017). Disruption of vHPC to PFC connections is also implicated in a variety of neuropsychiatric disor-

\footnotetext{
Received Feb. 9, 2018; revised May 22, 2018; accepted June 16, 2018.

Author contributions: X.L. and A.G.C. designed research; X.L. performed research; X.L. analyzed data; X.L. and A.G.C. wrote the paper.

This work was supported by NIH R01 MH085974 (A.G.C.). We thank the Carter laboratory for helpful discussions and comments on the manuscript, and Matthew Xu-Friedman for help with dynamic-clamp recordings.

The authors declare no competing financial interests.

Correspondence should be addressed to Dr. Adam G. Carter, Center for Neural Science, New York University,

4 Washington Place, New York, NY 10003. E-mail: adam.carter@nyu.edu.

DOI:10.1523/JNEUROSCI.0378-18.2018

Copyright $\odot 2018$ the authors $\quad 0270-6474 / 18 / 387351-13 \$ 15.00 / 0$
}

ders, including schizophrenia, stress and anxiety (Adhikari et al., 2010; Sigurdsson et al., 2010; Sigurdsson and Duvarci, 2015; Padilla-Coreano et al., 2016). However, although the importance of this circuit is broadly appreciated, relatively little is known about the cellular and synaptic properties of vHPC inputs to the PFC.

Long-range afferents can innervate multiple subregions of the PFC, including the prelimbic (PL) and infralimbic (IL) areas, which have different functional roles (Heidbreder and Groenewegen, 2003; Hoover and Vertes, 2007). Although there is no layer (L) 4 in the PFC, inputs can ramify through both superficial and deep layers to influence neural activity. A variety of projection neurons are also found in each cortical layer, which themselves send long-range outputs to other brain regions (Gabbott et al., 2005). For example, cortico-amygdala (CA) neurons in L2/3 project to basolateral amygdala (BLA), cortico-pontine (CP) neurons in $\mathrm{L} 5$ project via the pyramidal tract (PT), and corticocortical (CC) neurons across L2-L6 project to the contralateral PFC and other intratelencephalic targets (Hattox and Nelson, 2007; Dembrow et al., 2010; Morishima et al., 2011; Otsuka and Kawaguchi, 2011; Harris and Shepherd, 2015; McGarry and Carter, 2016; Anastasiades et al., 2018; Collins et al., 2018). Due to 
their locations in specific subregions and layers of the PFC, these projection neurons can sample distinct synaptic inputs (Little and Carter, 2012; McGarry and Carter, 2016; Anastasiades et al., 2018; Collins et al., 2018). Moreover, given their unique physiology, these neurons can differentially process their synaptic inputs to generate distinct patterns of action potential (AP) firing (Sheets et al., 2011; Anastasiades et al., 2018).

Recent studies indicate that long-range inputs to the PFC are often biased onto particular projection neurons, and this specificity can vary between PL and IL. BLA inputs are strongest at L2/3 CA neurons in PL (Little and Carter, 2013), but greatest at L5 PT neurons in IL (Cheriyan et al., 2016). In contrast, callosal inputs show no bias for different L2/3 pyramidal neurons (Little and Carter, 2013), but are stronger onto L5 PT neurons in PL (Anastasiades et al., 2018). vHPC afferents to the PFC are uniquely unidirectional, with no reciprocal projections from $\mathrm{PFC}$ back to the vHPC (Gabbott et al., 2005; Hoover and Vertes, 2007). Instead, vHPC inputs may preferentially target one or more projection neurons in the PFC to influence specific output pathways, and thereby regulate the top-down control of behavior (Euston et al., 2012). However, although in vivo studies have highlighted the functional importance of vHPC inputs for the encoding of both memory and emotional valence (Sotres-Bayon et al., 2012; Spellman et al., 2015; Padilla-Coreano et al., 2016), their ability to engage different PFC projection neurons is still unknown.

Here we combine optogenetics and whole-cell recordings in acute slices to study the cell-type specificity of vHPC to PFC connections in the mouse brain. We show that vHPC inputs target both IL and PL, but make distinct laminar connections in these adjacent subregions. We find that vHPC inputs make similar connections onto CC and CA neurons in superficial layers of IL. In contrast, vHPC inputs make preferential connections onto CC over CP neurons in deep layers of both IL and PL. We show that this connectivity leads to preferential recruitment of IL L5 CC neurons by vHPC inputs, even in the presence of feedforward inhibition. Finally, we establish that vHPC inputs facilitate at high frequencies, leading to enhanced activation of the cortical network. Together, our findings reveal the cell-type-specific organization of vHPC inputs, and provide a mechanistic description for how these connections can drive specific circuits in the PFC.

\section{Materials and Methods}

Animals. Experiments used wild-type mice of either sex in a C57BL/6J background (all purchased from The Jackson Laboratory). All experimental procedures were approved by the University Animal Welfare Committee of New York University.

Stereotaxic injections. Mice aged 4-6 weeks were deeply anesthetized with a mixture of ketamine and xylazine, and head fixed in a stereotax (Kopf Instruments). A small craniotomy was made over the injection site, using these coordinates relative to bregma: $\mathrm{PFC}= \pm 0.4,-2.3$, $+2.0 \mathrm{~mm}, \mathrm{BLA}=-3.1,-5,-1.2 \mathrm{~mm}$, Pons $=-0.6,-4.7,-4.0 \mathrm{~mm}$, mediodorsal thalamus $(\mathrm{MD})=-0.4,-3.6,-0.5 \mathrm{~mm}, \mathrm{vHPC}=-3.3$, both -3.6 and $4.2,-3 \mathrm{~mm}$ (mediolateral, dorsoventral, and rostrocaudal). For retrograde labeling, pipettes were filled with either red or green retrogradely transported beads (Lumafluor), cholera toxin subunit B (CTB) conjugated to either Alexa 488, 555, or 647 (Life Technologies), or RV- $\Delta$ G-mCherry (Salk). AAV1-CB7-CI-mCherry-WPRE-rBG (UPenn; AV-1-PV1969) was used for anterograde anatomy. For anterograde labeling of vHPC inputs for optogenetic experiments, pipettes were filled with AAV1-hSyn-hChR2(H134R)-EYFP (UPenn; AV-1-26973P). Borosilicate pipettes with 5-10 $\mu \mathrm{m}$ diameter tips were back-filled with dye and/or virus, a typical volume of $130-550 \mathrm{nl}$ was pressure-injected using a Nanoject III (Drummond) at 30 s intervals. The pipette was left in place for an additional $5 \mathrm{~min}$, allowing time to diffuse away from the pipette tip, before being slowly retracted from the brain. For retrobeads, CTB and AAV, animals were housed for $2-3$ weeks before recording. For RV, animals were housed for 7-10 d before imaging.

Slice preparation. Mice aged $6-8$ weeks were anesthetized with a lethal dose of ketamine and xylazine, and perfused intracardially with ice-cold external solution containing the following (in $\mathrm{mm}$ ): 65 sucrose, $76 \mathrm{NaCl}$, $25 \mathrm{NaHCO}_{3}, 1.4 \mathrm{NaH}_{2} \mathrm{PO}_{4}, 25$ glucose, $2.5 \mathrm{KCl}, 7 \mathrm{MgCl}_{2}, 0.4 \mathrm{Na}-$ ascorbate, and 2 Na-pyruvate (295-305 mOsm), and bubbled with $95 \%$ $\mathrm{O}_{2} / 5 \% \mathrm{CO}_{2}$. Coronal slices ( $300 \mu \mathrm{m}$ thick) were cut on a VS1200 vibratome (Leica) in ice-cold external solution, before being transferred to ACSF containing the following (in mM): $120 \mathrm{NaCl}, 25 \mathrm{NaHCO}_{3}, 1.4$ $\mathrm{NaH}_{2} \mathrm{PO}_{4}, 21$ glucose, $2.5 \mathrm{KCl}, 2 \mathrm{CaCl}_{2}, 1 \mathrm{MgCl}_{2}, 0.4 \mathrm{Na}$-ascorbate, and 2 Na-pyruvate (295-305 mOsm), bubbled with $95 \% \mathrm{O}_{2} / 5 \% \mathrm{CO}_{2}$. Slices were kept for $30 \mathrm{~min}$ at $35^{\circ} \mathrm{C}$, before being allowed to recover for $30 \mathrm{~min}$ at room temperature. All recordings were conducted at $30-32^{\circ} \mathrm{C}$.

Electrophysiology. Whole-cell recordings were obtained from pyramidal neurons located in specific layers of prelimbic and infralimbic PFC. Neurons were identified by infrared-differential interference contrast, as previously described (Chalifoux and Carter, 2010), and projection target was established by the presence of retrobeads or AlexaFluor-conjugated CTB under fluorescent illumination, as previously described (Little and Carter, 2013). Pairs of adjacent neurons were chosen for sequential recording, ensuring they received similar inputs (typically $<50 \mu \mathrm{m}$ between cells). Borosilicate pipettes (2-5 M $\Omega$ ) were filled with one of two internal solutions. For current-clamp (in $\mathrm{mm}$ ): $135 \mathrm{~K}$-gluconate, $7 \mathrm{KCl}$, 10 HEPES, $10 \mathrm{Na}$-phosphocreatine, $4 \mathrm{Mg}_{2}$-ATP, and $0.4 \mathrm{Na}-\mathrm{GTP}, 290-$ 295 mOsm, pH 7.3, with KOH. For voltage-clamp (in mM): 135 Csgluconate, 10 HEPES, 10 Na-phosphocreatine, $4 \mathrm{Mg}_{2}$-ATP, and 0.4 Na-GTP, 0.5 EGTA, 10 TEA-chloride, and 2 QX314, 290-295 mOsm, $\mathrm{pH} 7.3$, with $\mathrm{CsOH}$. In some experiments involving two-photon imaging, $30 \mu \mathrm{M}$ AlexaFluor 594 was included to allow morphological reconstruction of dendrites. Fluorescent dye was allowed to diffuse throughout the dendritic arbor for at least 20 min before imaging cell morphology.

Electrophysiology recordings were made with a MultiClamp 700B amplifier (Molecular Devices), filtered at $4 \mathrm{kHz}$ for current-clamp and $2 \mathrm{kHz}$ for voltage-clamp, and sampled at $10 \mathrm{kHz}$. The initial series resistance was $<20 \mathrm{M} \Omega$, and recordings where ended if series resistance rose $>25 \mathrm{M} \Omega$. For voltage-clamp recordings, EPSCs and IPSCs were recorded at $-70 \mathrm{mV}$ and $+10 \mathrm{mV}$, respectively. In some experiments, $1 \mu \mathrm{M}$ TTX was added to block APs, along with $100 \mu \mathrm{M} 4-\mathrm{AP}$ and $4 \mathrm{~mm}$ external $\mathrm{Ca}^{2+}$ to restore presynaptic release. In many experiments, $10 \mu \mathrm{M} C P P$ was used to block NMDA receptors. In dynamic-clamp experiments, $10 \mu \mathrm{M}$ NBQX, $10 \mu \mathrm{M}$ CPP and $10 \mu \mathrm{M}$ gabazine were used to block both excitation and inhibitory synaptic transmission. All chemicals were purchased from either Sigma-Aldrich or Tocris Bioscience.

Dynamic-clamp recordings. Dynamic-clamp recordings were performed using an ITC-18 interface (Heka Electronics) with Igor Pro (WaveMetrics) running MafPC (courtesy of Matthew Xu-Friedman, University at Buffalo, SUNY; Carter and Regehr, 2002). Experimentally recorded EPSCs were first converted into excitatory postsynaptic conductances (EPSGs) by dividing by the driving force. These conductances were then "injected" into neurons, with the dynamic-clamp updating current injections based on voltage recordings at $50 \mathrm{kHz}$. The reversal potential for excitation was set to the experimentally measured value of $+10 \mathrm{mV}$. Conductances were multiplied by a range of scale factors (1$10 \times$ ) to mimic increasing activity, with unit scale factor of IL L5 CC cell corresponding to $3.5 \mathrm{nS}$ conductance (Anastasiades et al., 2018).

Optogenetics. Channelrhodopsin-2 (ChR2) was expressed in presynaptic neurons, and activated with a brief light pulse from a blue LED (473 nm; Thorlabs). For wide-field illumination, light was transmitted via a $10 \times 0.3$ NA objective (Olympus) centered $350 \mu \mathrm{m}$ from the midline. For subcellular mapping, a $60 \times 1.0$ NA objective (Olympus) was focused on the dendrites (Collins et al., 2018). The aperture was minimized to reach an effective diameter of $\sim 50 \mu \mathrm{m}$. Maximum LED power was measured as $10 \mathrm{~mW}$ at the back aperture of the objective. For each 
A

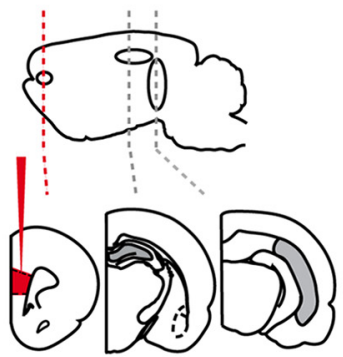

PFC dHPC VHPC RV-mCherry
PFC

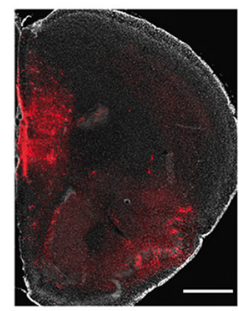

B

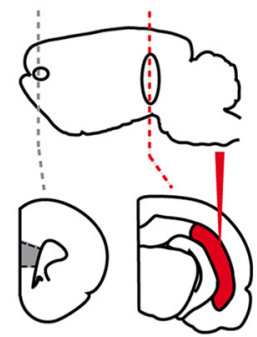

PFC vHPC AAV-mCherry
vHPC

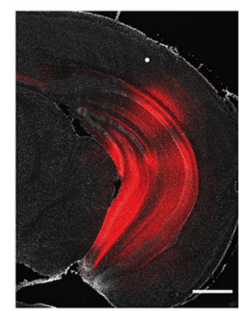

PFC

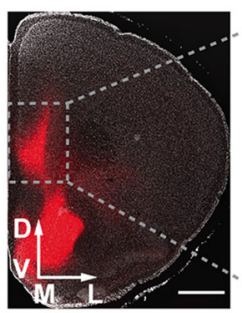

vHPC
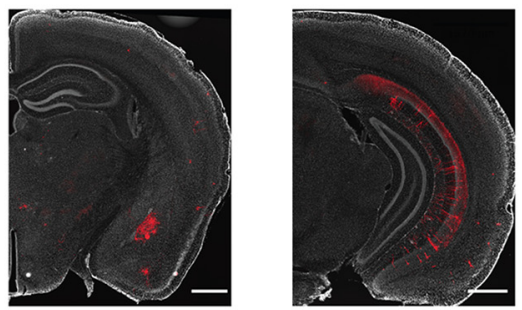

L1 $2 / 3 \quad 5 \quad 6$

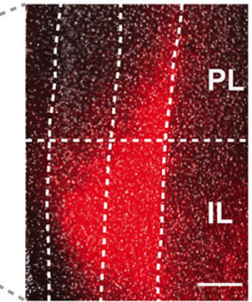

\section{C}
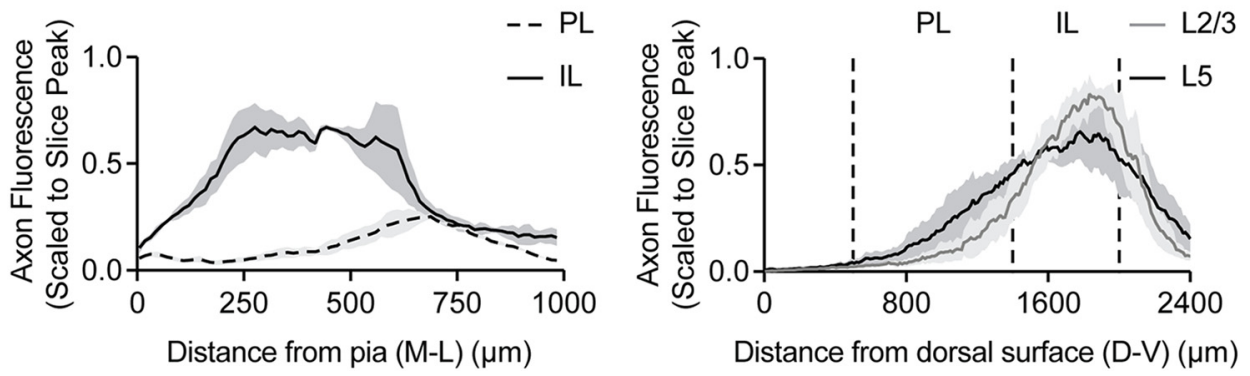

Figure 1. VHPC projects to the infralimbic and prelimbic PFC. A, Left, RV-mCherry injection into the PFC. Right, Fluorescent images of injection site (PFC), lack of retrograde labeling in dHPC, and prominent retrograde labeling in vHPC. Red, mCherry; gray, DAPI. Scale bars, $800 \mu \mathrm{m}$. B, Left, AAV-mCherry injection into the vHPC. Right, Fluorescent images of injection site (vHPC) and prominent anterograde labeling in PFC (bregma $+2.0 \mathrm{~mm}$; scale bars, $800 \mu \mathrm{m}$ ) and expanded view of IL ( $1400-2000 \mu \mathrm{m}$ from dorsal surface) and PL ( $500-1400 \mu \mathrm{m}$ from dorsal surface) regions. Scale bar, $200 \mu \mathrm{m}$. Red, mCherry; gray, DAPI. C, Left, vHPC axon distribution in PL (measured at 900-1200 $\mu \mathrm{m}$ from dorsal surface) and IL (measured at $1600 \mu \mathrm{m}-1900 \mu \mathrm{m}$ from dorsal surface), as a function of the distance from the midline pia along the medial-lateral axis. Right, vHPC axon distribution along dorsal-ventral axis in L2/3 (measured at $250-350 \mu \mathrm{m}$ from pia) and L5 (measured at $400-500 \mu \mathrm{m}$ from pia) of IL and PL regions, as a function of the distance from the dorsal surface along the dorsal-ventral axis. Plots show axon fluorescence normalized to the peak fluorescence within the slice ( $n=3$ animals).

experiment, LED pulse power and duration were adjusted to obtain reliable responses, with typical values of $0.4-10 \mathrm{~mW}$ and $3 \mathrm{~ms}$, respectively.

Two-photon microscopy. Two-photon imaging was performed on a custom microscope, as previously described (Chalifoux and Carter, 2010). A Ti:Sapphire laser (Coherent) at $810 \mathrm{~nm}$ was used to excite AlexaFluor 594 to image morphology with a $60 \times 1.0$ NA objective (Olympus).

Histology and fluorescence microscopy. Mice were anesthetized with a lethal dose of ketamine and xylazine and perfused intracardially with $0.01 \mathrm{M}$ PBS followed by $4 \%$ paraformaldehyde (PFA) in $0.01 \mathrm{M}$ PBS. Brains were fixed in $4 \%$ PFA in $0.01 \mathrm{M}$ PBS overnight at $4^{\circ} \mathrm{C}$. Slices were prepared at a thickness of $70 \mu \mathrm{m}$ (Leica VT 1000S vibratome) and mounted under glass coverslips on gelatin-coated slides using ProLong Gold antifade reagent with DAPI (Invitrogen). Whole-brain images were acquired using a slide-scanning microscope (Olympus VS120) with a $10 \times 0.25 \mathrm{NA}$ or $20 \times 0.75 \mathrm{NA}$ objective. Image processing involved adjusting brightness, contrast, and quantifying fluorescence level using ImageJ (NIH).

Data analysis. Electrophysiology and imaging data were acquired using National Instruments boards and custom software in MATLAB
(MathWorks). Off-line analysis was performed using Igor Pro (WaveMetrics). PSC amplitudes were measured as the average value across $1 \mathrm{~ms}$ around the peak. PSC charge transfers were measured as the area between PSC trace and baseline. For axon fluorescence analysis in the PFC, fluorescence intensity along a selected axis was quantified using ImageJ. For each trace of axon fluorescence, values were scaled to the peak fluorescence within the slice. Scaled traces collected from three animals were used to plot averages \pm SEM. For colocalization analysis in PFC, cell counting was performed in ImageJ on a multicolor image of retrogradely labeled neurons with DAPI labeling. Labeled cell bodies were manually counted in regions $300 \times 900 \mu \mathrm{m}$ in PFC. Distance from the pial surface was used to sort cells into $50 \mu \mathrm{m}$ bins. The number of cells per bin was averaged across three slices from each animal, and these average values were used to calculate averages \pm SEM across animals. Reconstruction of dendritic morphology was performed using Neurolucida 360 (MBF Bioscience).

Experimental design and statistical analysis. Each dataset was collected from at least three animals. For any physiology experiment, a total number of at least seven cells, pairs or triplets are sampled. Statistical analysis is performed using Prism 7.0 (GraphPad). Summary data of raw values 
are reported in the text and figures as arithmetic mean \pm SEM, ratios are reported as geometric mean in the text, and with $\pm 95 \%$ confidence interval in the figures, unless otherwise noted. Comparisons between unpaired data were performed using unpaired $t$ tests. Comparisons between data recorded in pairs were performed using ratio-paired $t$ tests. Comparisons of values across multiple variables were performed using two-way ANOVA, with Sidak's test for multiple comparisons. Two-tailed $p$ values $<0.05$ were considered significant.

\section{Results}

\section{vHPC projects to the IL and PL PFC}

We initially used anatomical approaches to determine which part of the HPC projects to the medial PFC. We first injected an mCherry-expressing rabies virus (RVmCherry) into the PFC and waited 7-10 d for retrograde expression (Fig. 1A). We observed minimal labeling of dorsal HPC (dHPC), but prominent labeling across the dorsal-ventral axis of the vHPC. To confirm that neurons in $\mathrm{VHPC}$ project to the PFC, we then injected an mCherryexpressing adeno-associated virus (AAVmCherry) into the vHPC and waited $14-21 \mathrm{~d}$ for expression (Fig. 1B). We observed red fluorescent axons in both the IL and PL regions of the PFC, which was especially robust in IL, consistent with a direct projection. Quantifying axon density profiles in IL and PL showed distinct projection patterns, with vHPC inputs to the superficial and deep layers of IL, but only to deep layers of PL (Fig. $1 C ; n=3$ animals). These findings indicate that the hippocampal-prefrontal projection primarily originates from the vHPC, and may differentially influence IL and PL.

\section{vHPC contacts superficial and deep pyramidal neurons}

We next used optogenetics and whole-cell recordings to examine connections made by vHPC inputs at pyramidal neurons in the PFC. We injected ChR2-expressing virus (AAV-ChR2-EYFP) into the vHPC, waited 2-3 weeks for expression, and prepared ex vivo slices of medial PFC (Fig. 2A). We recorded from pyramidal neurons whose cell bodies were located in L2/3, L5, or L6 of IL or PL (Fig. $2 A)$. In dendritic reconstructions from two-photon images, we found these neurons have characteristically distinct morphologies, with L2/3 neurons sampling inputs to superficial layers, L6 neurons sampling primarily deep layers, and L5 neurons sampling both. We activated vHPC inputs using wide-field illumination and compared synaptic responses at sequentially recorded pairs or triplets of neurons in the same slice of PFC, with all comparisons including a L5 neuron. To isolate monosynaptic inputs, we included TTX $(1 \mu \mathrm{M}), 4$-AP $(100 \mu \mathrm{M})$, and elevated $\mathrm{Ca}^{2+}(4 \mathrm{~mm})$, which blocks APs but restores presynaptic release (Petreanu et al., 2009; Little and Carter, 2012). We kept light intensity and duration constant within pairs or triplets in the same slice, which allowed us to account for variability in viral expression across animals and slices (Little and Carter, 2013; McGarry and Carter, 2016; Anastasiades et al., 2018; Collins et al., 2018). vHPC inputs evoked pronounced excitatory postsynaptic currents (EPSCs) at a holding potential of $-70 \mathrm{mV}$ at cells located in both IL and PL. Within IL, we found robust EPSCs in both L2/3 and L5 neurons, but negligible responses in L6 neurons (Fig. $2 B ; \mathrm{L} 2 / 3=681 \pm 188 \mathrm{pA}, \mathrm{L} 5=647 \pm 83 \mathrm{pA} ; \mathrm{L} 6=49 \pm 14 \mathrm{pA}$; $\mathrm{L} 2 / 3 / \mathrm{L} 5$ ratio $=0.89, p=0.7 ; \mathrm{L} 6 / \mathrm{L} 5$ ratio $=0.05, p=0.005$, ratiopaired $t$ tests; $n=7$ pairs each, 6 animals). In contrast, we observed that EPSCs were strongly biased toward L5 neurons in PL, with minimal responses in both $\mathrm{L} 2 / 3$ and $\mathrm{L} 6$ neurons (Fig. $2 C$; $\mathrm{L} 2 / 3=5 \pm$ $3 \mathrm{pA}, \mathrm{L} 5=375 \pm 91 \mathrm{pA}, \mathrm{L} 6=69 \pm 34 \mathrm{pA} ; \mathrm{L} 2 / 3 / \mathrm{L} 5$ ratio $=0.01, p=$ $0.0001 ; \mathrm{L} 6 / \mathrm{L} 5$ ratio $=0.10, p=0.03$, ratio-paired $t$ tests; $n=7$ pairs each, 6 animals). Together, these findings indicate that vHPC targets three different areas of the PFC, with sizeable synaptic responses in both superficial and deep IL, but only in deep PL. 
A

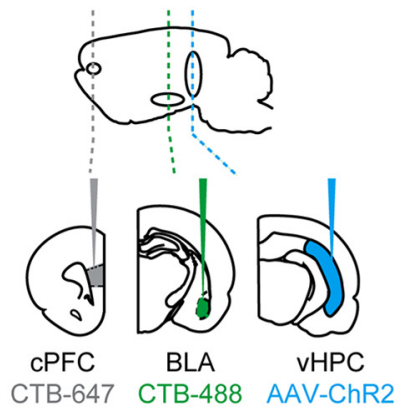

B

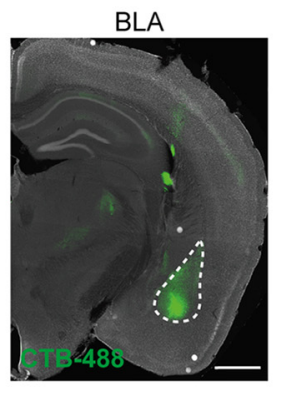

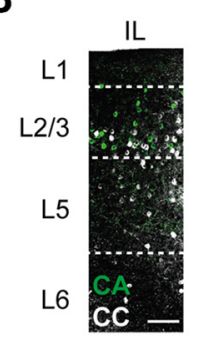

D
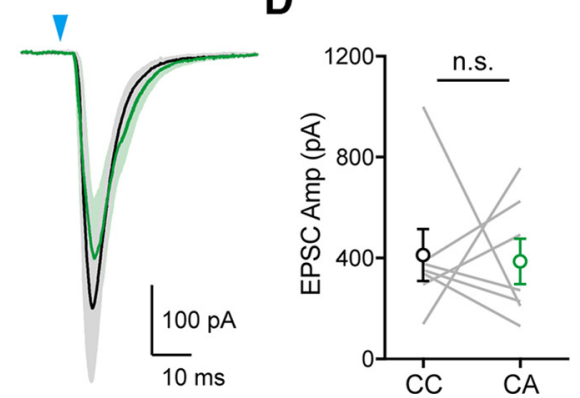

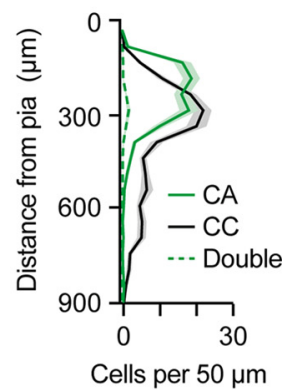

C

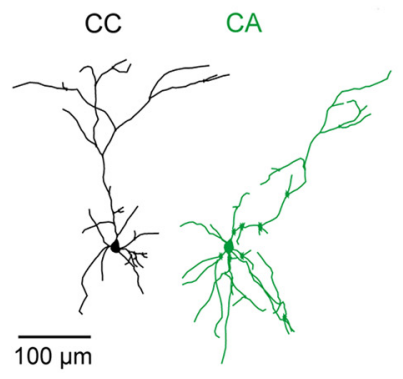

Figure 3. VHPC inputs contact L2/3 CC and CA neurons in IL PFC. A, Left, AAV-ChR2 injection into the vHPC, and simultaneous injection of CTB-488 or -647 into the BLA and CPFC. Right, Example fluorescent image of injection sites in the BLA. Scale bar, $800 \mu \mathrm{m}$. B, Left, Distribution of CC (white) and CA (green) neurons in the IL PFC. Scale bar, $100 \mu \mathrm{m}$. Right, Summary of CC and CA neuron distribution in IL. C, Left, Reconstructed dendritic morphologies of CC and CA neurons. Scale bar, $100 \mu \mathrm{m}$. Right, Average vHPC-evoked EPSCs at $-70 \mathrm{mV}$ from pairs of L2/3 CC (black) and L2/3 CA (green) neurons in IL. Blue arrow $=3$ ms light stimulation. D, Left, Summary of EPSC amplitudes from recordings in C. Right, Summary of EPSC amplitude ratios ( $n=7$ pairs, 4 animals). Note the logarithmic axis.

\section{vHPC equally targets CC and CA neurons in superficial IL PFC}

Hippocampal afferents to superficial IL are thought to be important for engaging fear-related behaviors (Marek et al., 2018). Other long-range inputs can target specific classes of $\mathrm{L} 2 / 3$ projection neurons, with BLA inputs biased onto CA neurons (Little and Carter, 2013; McGarry and Carter, 2016). To examine whether vHPC inputs show similar specificity, we first coinjected different colors of retrogradely transported, fluorescently-tagged CTB into the BLA and contralateral PFC (cPFC) of the same animals (Fig. 3A). We observed minimal overlap between CC and CA neurons in superficial layers of IL, indicating that these cells represent distinct populations (Fig. 3B; $2.0 \pm 0.4 \%$ colabeling, $n=4$ animals, 3 slices per animal). To examine synaptic responses, we then repeated these retrograde tracer injections, while also coinjecting AAV-ChR2 into the vHPC. Making sequential recordings from neighboring pairs of CA and CC neurons within the same slice (Fig. $3 C$ ), we observed that vHPC inputs evoked prominent EPSCs recorded at $-70 \mathrm{mV}$ in both cell types (Fig. 3C). Interestingly, response amplitudes were similar at $\mathrm{CA}$ and CC neurons (Fig. $3 D$; $\mathrm{CA}=388 \pm 90 \mathrm{pA}, \mathrm{CC}=412 \pm$ $103 \mathrm{pA}$; CA-CC ratio $=0.92, p=0.84$, ratio-paired $t$ test; $n=7$ pairs, 4 animals), without the bias previously seen for BLA inputs onto CA neurons (Little and Carter, 2013; McGarry and Carter, 2016). These findings show that vHPC inputs make equivalent connections onto neurons projecting to the $\mathrm{PFC}$ or $\mathrm{BLA}$, and are thus capable of engaging both networks.

\section{vHPC preferentially targets CC neurons in deep IL and PL PFC}

Our initial experiments indicated that vHPC inputs also make prominent connections onto L5 pyramidal neurons in both IL and PL. Cortical L5 contains CC and CP neurons, which are spatially intermingled but distinct populations (Harris and Shepherd, 2015). Previous studies have shown that BLA and callosal inputs are strongest onto $\mathrm{CP}$ and other pyramidal tract neurons in L5 of IL and PL, respectively (Cheriyan et al., 2016; Anastasiades et al., 2018). However, whether vHPC inputs preferentially contact CC or CP neurons in L5 of these two subregions remains unclear. To examine this targeting, we first coinjected separate CTBs into the $\mathrm{PFC}$ and ipsilateral pons of the same animals (Fig. $4 A$ ). We observed minimal overlap between CC and CP neurons in either IL or PL, confirming that they are distinct cell types (Fig. $4 B$; colabeling: $\mathrm{PL}=0.20 \pm 0.17 \%$, $\mathrm{IL}=0.04 \pm 0.04 \%, n=3$ animals, 3 slices per animal). In reconstructions from twophoton images, we also found that CC neurons have much sparser dendrites in both IL and PL (Fig. 4C,D), consistent with previous observations that $\mathrm{CP}$ neurons possess denser apical dendrites than CC neurons (Hattox and Nelson, 2007; Dembrow et al., 2010; Avesar and Gulledge, 2012; Anastasiades et al., 2018; Collins et al., 2018). To examine synaptic responses, we next coinjected AAV-ChR2 into the vHPC of the same animals, and sequentially recorded from pairs of adjacent CC and CP neurons in the same slice. We found that vHPC inputs evoked EPSCs at $-70 \mathrm{mV}$ in both cell types and regions of the PFC (Fig. 4C,D; IL CC $=862 \pm 92 \mathrm{pA}, \mathrm{IL} \mathrm{CP}=615 \pm 122 \mathrm{pA}, \mathrm{PL}$ $\mathrm{CC}=665 \pm 89 \mathrm{pA}, \mathrm{PLCP}=198 \pm 45 \mathrm{pA})$. However, response amplitudes were larger at CC neurons, with a slight bias in IL $(\mathrm{CP} / \mathrm{CC}$ ratio $=0.61, p=0.035$, ratio-paired $t$ test; $n=13$ pairs, 5 animals), and strong bias in PL (CP/CC ratio $=0.17$, $p=0.007$, ratio-paired $t$ test; $n=10$ pairs, 5 animals; Fig. $4 E-G)$. Thus, CC neurons receive much stronger inputs from the vHPC than neighboring CP neurons, indicating that vHPC inputs to deep layers of PFC preferentially contact neurons that project within the cortex. 
A

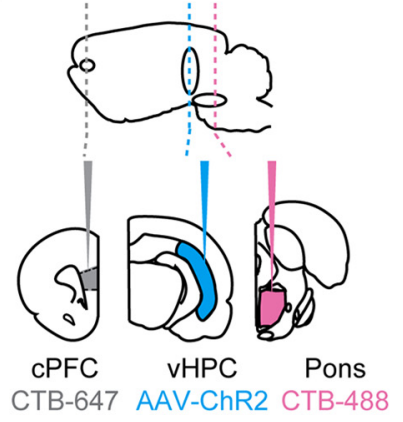

C

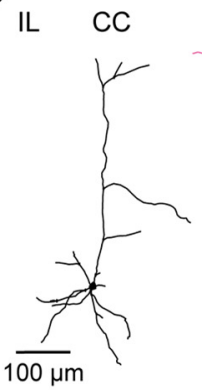

E
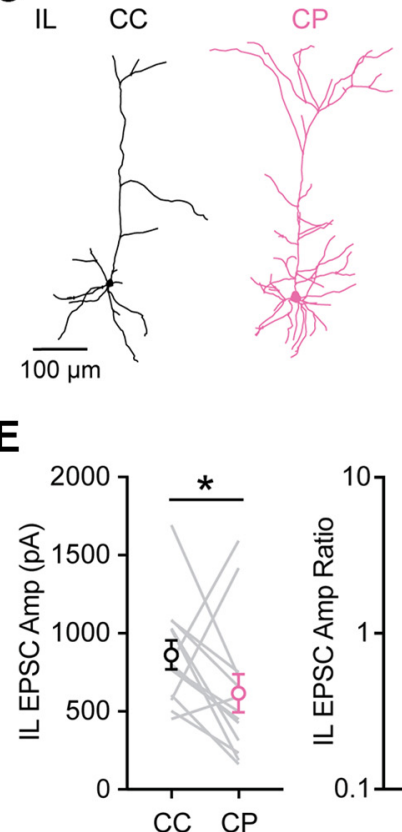

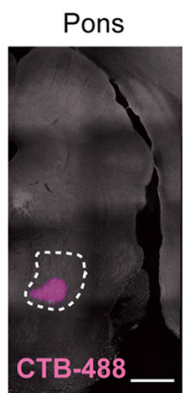

PL

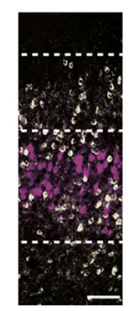

PL

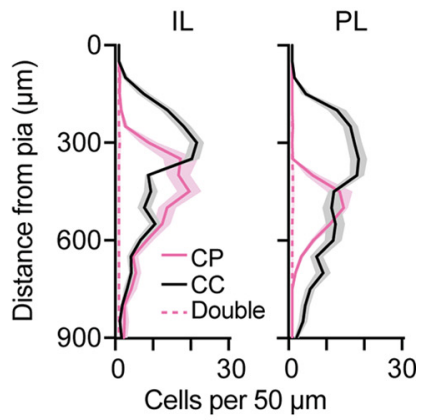

D
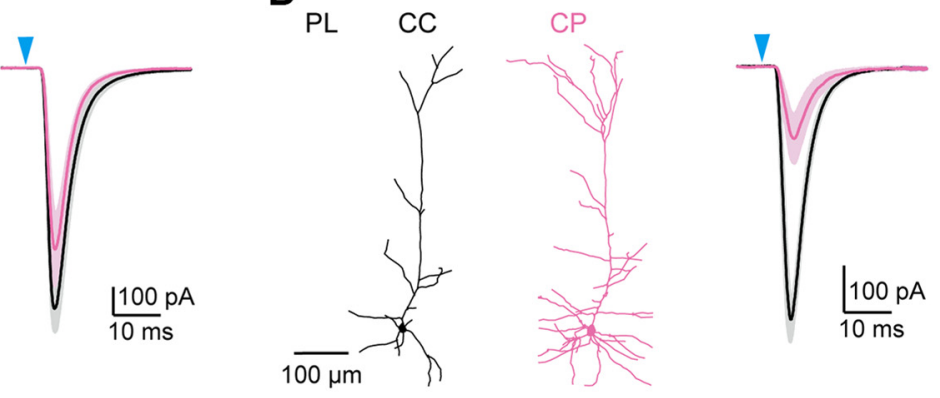

F
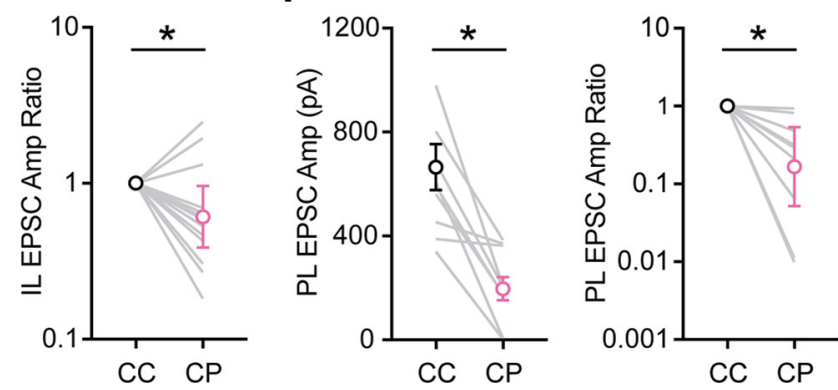

G

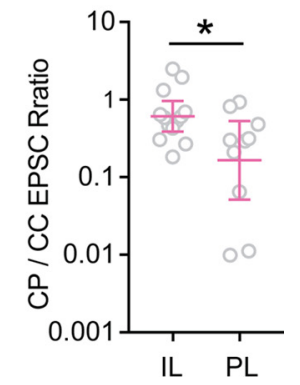

Figure 4. vHPC inputs target L5CC neurons in both IL and PL PFC. A, Left, AAV-ChR2 injection into the vHPC, and simultaneous injection of CTB-488 or -647 into the pons and CPFC. Right, Example confocal image of injection sites in the pons. Scale bar, $600 \mu \mathrm{m}$. B, Left, Distribution of CC (white) and CP (purple) neurons in the IL and PL PFC. Scale bar, $100 \mu \mathrm{m}$. Right, Summary of CC and CP neuron distributions in IL and PL. C, Left, Reconstructed dendritic morphologies of L5 CC and CP neurons in IL. Right, Average vHPC-evoked EPSCs at $-70 \mathrm{mV}$ from pairs of L5 CC (black) and L5 CP (purple) neurons in IL ( $n=13$ pairs, 5 animals). Blue arrow $=3$ ms light stimulation. $D$, Similar to C for L5 CC and CP neurons in PL ( $n=10$ pairs, 5 animals). E, Left, Summary of EPSC amplitudes from $\boldsymbol{C}$. Right, Summary of EPSC amplitude ratios from $\boldsymbol{C}$. Note the logarithmic axis. $\boldsymbol{F}$, Similar to $E$ for $L 5 C C$ and $C P$ neurons in PL, summarized from $\boldsymbol{D}$. G, Summary of CC/CP EPSC amplitude ratios from recordings in $\boldsymbol{E}$ and $\boldsymbol{F}$ between pairs of cells in PL and IL. Note the logarithmic axis. ${ }^{*} p<0.05$.

Dendrites of L5 neurons differentially sample vHPC inputs While vHPC axons ramify throughout superficial and deep layers of IL, they are primarily restricted to deep layers of PL. Therefore, L5 pyramidal neurons may sample both superficial and deep vHPC inputs in IL, but only deep vHPC inputs in PL. Moreover, the larger apical dendrites of L5 CP neurons may allow them to sample more superficial vHPC inputs relative to L5 CC neurons (Anastasiades et al., 2018; Collins et al., 2018). By targeting different dendritic compartments, long-range inputs can evoke distinct functional responses at different L5 neurons (Dembrow et al., 2015). To examine dendritic targeting of vHPC inputs at defined PFC cell types, we cycled a focused light beam across multiple dendritic locations, eliciting spatially restricted EPSCs (Petreanu et al., 2009; Fig. 5A). Although these somatic recordings do not compensate for dendritic filtering, they can reveal the targeting of a given input in the dendrites. For example, callosal inputs primarily target the proximal dendrites of L5 CC and cortico-thalamic (CT) neurons (Anastasiades et al., 2018), whereas thalamic inputs preferentially target apical dendrites of L5 CT neurons (Collins et al., 2018). For vHPC inputs, we found synaptic responses were strongest near the soma and proximal dendrites and diminished toward the distal dendrites for both L5 CC and CP neurons in PL and IL (Fig. $5 B ; n=7$ cells each, 3 animals each). Interestingly, vHPC inputs targeted the proximal dendrites more in CP neurons than CC neurons, and this bias was greater in IL (Fig. 5B-D; arithmetic mean of charge ratio $(+200$ $\mu \mathrm{m}$ dendrite/soma) in IL: $\mathrm{CC}=0.31, \mathrm{CP}=1.17, p=0.026$, unpaired $t$ test; $n=7$ cells, 3 animals each). These findings suggest that the larger dendrites of CP neurons allow them to better sample the superficial vHPC inputs to IL, although most of these connections remain close to the soma.

\section{vHPC inputs weakly target CT neurons in both L5 and L6 of IL PFC}

Our initial experiments also showed that vHPC inputs are relatively weak in L6 of the PFC, which is enriched in CT neurons that project to the thalamus (Collins et al., 2018). To further explore how vHPC inputs influence these neurons, we next coinjected fluorescently-tagged, retrogradely transported beads and CTB into the MD and CPFC, along with AAV-ChR2 into the vHPC of 
A

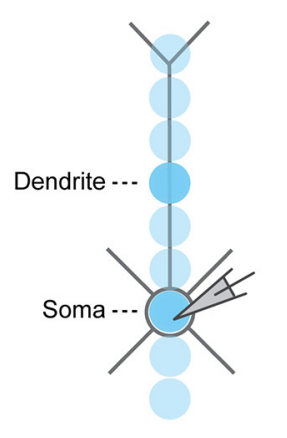

B

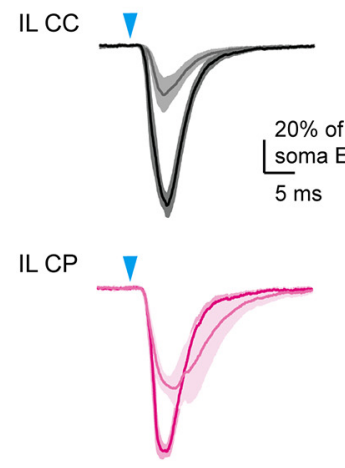

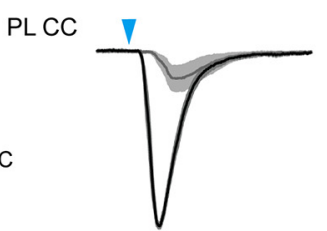

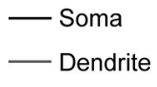

PLCP

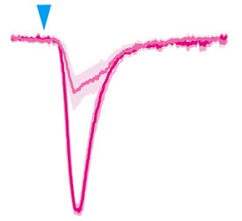

\section{— Soma
— Dendrite}

D
C

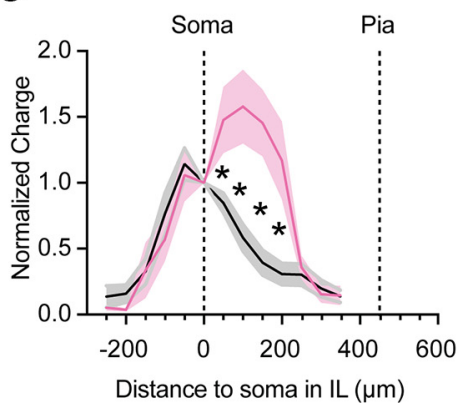

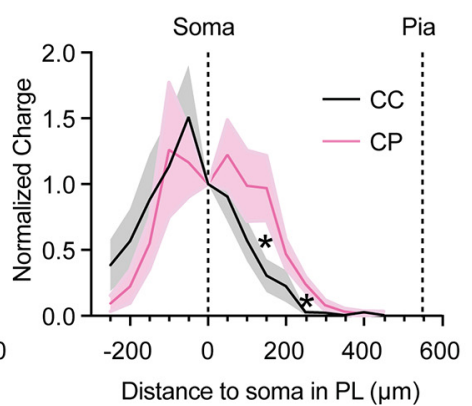

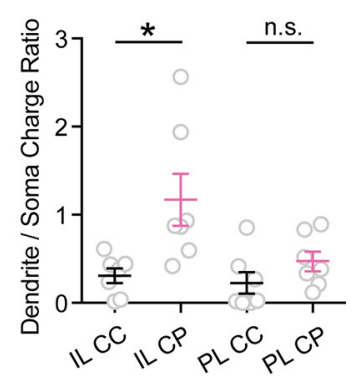

Figure 5. VHPC inputs differentially contact the dendrites of $L 5$ neurons. $A$, Recording schematic. Focused light was delivered along the main apical dendrite of the recorded cell, with $50 \mu \mathrm{m}$ increments between stimulation spots. Darker spots represent the soma and a location $+200 \mu \mathrm{m}$ along the dendrite, as shown in $\boldsymbol{B}$. $\boldsymbol{B}$, Average vHPC-evoked EPSCs recorded at $-70 \mathrm{mV}$ when stimulating at either the soma (dark) or apical dendrites ( $+200 \mu \mathrm{m}$ from the soma; light) in L5 CC (black) and L5 CP (purple) neurons in either IL or PL. For each cell, EPSCs were normalized to the response evoked from stimulation of the soma. Blue arrow $=3 \mathrm{~ms}$ light stimulation ( $n=7$ cells, 3 animals for each cell type). C, Summary of EPSC charges (arithmetic mean and SEM, normalized to the responses evoked at soma) measured at different distances from the soma in L5CC (black) or L5 CP (purple) neurons in either IL (left) or PL (right). D, Ratios of EPSC charges evoked in proximal apical dendrites $(+200 \mu \mathrm{m})$ and soma. Individual values, arithmetic mean, and SEM are plotted for each cell type. ${ }^{*} p<0.05$.

the same animal (Fig. 6A). We then examined synaptic responses at sequentially recorded triplets of CC and CT neurons in L5 and L6 of the same slice. Dendritic reconstructions showed that both L5 and L6 CT neurons have extended dendrites that are positioned to sample the hippocampal axonal field (Fig. 6B). However, when we stimulated $\mathrm{vHPC}$ inputs and recorded at $-70 \mathrm{mV}$, we observed large EPSCs at L5 CC neurons, smaller responses at L5 CT neurons, and negligible responses at L6 CT neurons (Fig. $6 \mathrm{~B}, \mathrm{C}$; L5 CC $=529 \pm 43 \mathrm{pA}, \mathrm{L} 5 \mathrm{CT}=296 \pm 57 \mathrm{pA}, \mathrm{L} 6 \mathrm{CT}=$ $57 \pm 10 \mathrm{pA} ; \mathrm{L} 5 \mathrm{CT} / \mathrm{CC}$ ratio $=0.50, p=0.02 ; \mathrm{L} 6 \mathrm{CT} / \mathrm{L} 5 \mathrm{CC}$ ratio $=0.10, p<0.0001$, ratio-paired $t$ tests, $n=8$ triplets, 5 animals). Together, these results indicate that vHPC inputs have limited monosynaptic access to the corticothalamic pathway emerging from L5 and L6 CT neurons of the PFC.

\section{vHPC preferentially activates deep CC neurons in IL PFC}

Our results suggest that vHPC inputs make particularly strong connections onto CC neurons in multiple parts of the PFC. To determine the primary target, we next compared vHPC-evoked EPSCs in sequential triplets of IL L2/3 CC, IL L5 CC and PL L5 CC neurons in the same slice. The strongest EPSCs were observed at IL L5, followed by IL L2/3, with much weaker responses at PL L5 (Fig. 7A). Comparing the ratio of responses confirmed that IL L5 $\mathrm{CC}$ neurons are the primary recipient of $\mathrm{vHPC}$ inputs (IL L2/3 CC/IL L5 CC ratio $=0.63, p=0.03$; PL L5 CC/IL L5 CC ratio = $0.26, p=0.004 ; n=10$ triplets, 5 animals). We next used these response ratios to normalize EPSC strength across the six celltypes recorded in IL and PL (Fig. 7B). For example, to compare responses at IL L5 CC neurons with those at PL L5 CP neurons, we multiplied the (PL L5 CP/PL L5 CC) ratio by the (PL L5 CC/IL L5 CC) ratio. This provided a hierarchy of the relative input strengths, with strongest inputs onto IL L5 CC neurons and weakest onto PL L5 CP neurons.

Having established the targeting of vHPC inputs onto PFC projection neurons, we sought to determine which is most strongly driven to fire APs. Postsynaptic responses reflect both synaptic input and the intrinsic properties of postsynaptic neurons, which also depend on projection class. For example, superficial CC and CA neurons have indistinguishable intrinsic properties and excitability (Little and Carter, 2013). In contrast, L5 CC neurons have a higher input resistance than L5 CP neurons, and lack appreciable h-current, making them much more excitable (Dembrow et al., 2010; Sheets et al., 2011; Anastasiades et al., 2018). To assess AP firing without triggering network activity, we used dynamic-clamp recordings to inject synaptic conductances into specific cells (Carter and Regehr, 2002; Dembrow et al., 2015; Anastasiades et al., 2018). We first converted the normalized EPSCs into conductances, and then multiplied them by a range of linear scale factors (Fig. $7 C$ ). We then injected these conductances into their respective neuronal subtypes, evoking subthreshold EPSPs at lower scale factors and suprathreshold APs at higher scale factors (Fig. 7C). Quantifying AP probability as a function of scale factor, we established the order of firing in the six cell types (Fig. 7D), with strongest activation of IL L5 CC (IL L5 CC vs other cell types, scale factor $\times$ cell type interaction: $F_{(45,380)}=4.15, p<0.0001$, two-way ANOVA; $n=7$ cells, 3 animals for each cell type), followed by weaker activation of IL $\mathrm{L} 2 / 3 \mathrm{CC}$ and CA, and minimal activation of CP and CC neurons 
in PL. Together, these findings predict that the cell-type-specific targeting translates to the preferential activation of IL L5 CC neurons over all other projection neurons.

\section{Activation of IL L5 CC neurons persists with inhibition}

Our dynamic-clamp recordings suggested that IL L5 CC neurons are most strongly activated by vHPC inputs. However, vHPC inputs can also engage inhibitory interneurons in PFC (Marek et al., 2018), which could prevent evoked AP firing (Pouille and Scanziani, 2001; Tierney et al., 2004; Isaacson and Scanziani, 2011). We next assessed the extent to which vHPC inputs engage feedforward inhibition at the three types of CC neurons. We used similar viral and labeling approaches, but in this case conducted experiments in the absence of TTX and 4-AP, to allow for feedforward inhibition in the network. In sequential recordings from triplets of IL L2/3, IL L5 and PL L5 in the same slice, we observed pronounced vHPC-evoked EPSCs in CC neurons at $-70 \mathrm{mV}$ and inhibitory postsynaptic currents (IPSCs) at $+10 \mathrm{mV}$ (Fig. 8A). Both EPSCs and IPSCs were strongest in IL L5 CC neurons (Fig. 8B; EPSCs: IL L2/3/IL L5 ratio $=0.49, p=0.012 ;$ PL L5/IL L5 ratio $=$ $0.24, p=0.0003$; IPSCs: IL L2/3/IL L5 ratio $=0.73, p=0.16 ;$ PL L5/IL L5 ratio $=$ $0.33, p=0.004$, ratio-paired $t$ tests; $n=11$ triplets, 6 animals), mirroring our findings with isolated monosynaptic EPSCs. Interestingly, the EPSC/IPSC (E/I) ratio was similar across the three cell types, suggesting balanced excitation and inhibition (Fig. 8B). To assess the functional consequences of this targeting, we examined vHPC-evoked EPSPs and AP firing at triplets of CC neurons (Fig. 8C). Consistent with our earlier results, we found that vHPC most strongly activated IL L5 CC neurons (Fig. $8 D$; AP probability: IL L2/3 = $0.04 \pm 0.04$, IL L5 $=0.72 \pm 0.11$, PL L5 $=0$; IL L5 vs IL L2/3, $p=$ 0.0005 ; IL L5 vs PL L5, $p=0.0006$, paired $t$ tests; $n=7$ triplets, 4 animals), indicating preferential activation even in the presence of feedforward inhibition.

\section{Facilitating vHPC activation is frequency dependent}

While much of our analysis used single stimuli, hippocampal neurons projecting to PFC fire repetitively in behaving animals (Siapas et al., 2005). In addition, repetitive trains of optogenetic stimuli are commonly used to assess functional connections in vivo (Do-Monte et al., 2015; Kitamura et al., 2017; Roy et al., 2017). During repetitive activity, short-term synaptic dynamics could strengthen or weaken vHPC inputs (Zucker and Regehr, 2002). Moreover, feedforward inhibition can change during repetitive activity, depending in part on the types of interneurons that are involved (Cruikshank et al., 2012; McGarry and Carter, 2016; Tremblay et al., 2016). Therefore, to study the sustained influence of vHPC projections during repetitive activity, we next examined excitation and feedforward inhibition evoked by trains of vHPC inputs at
$\mathbf{B}$
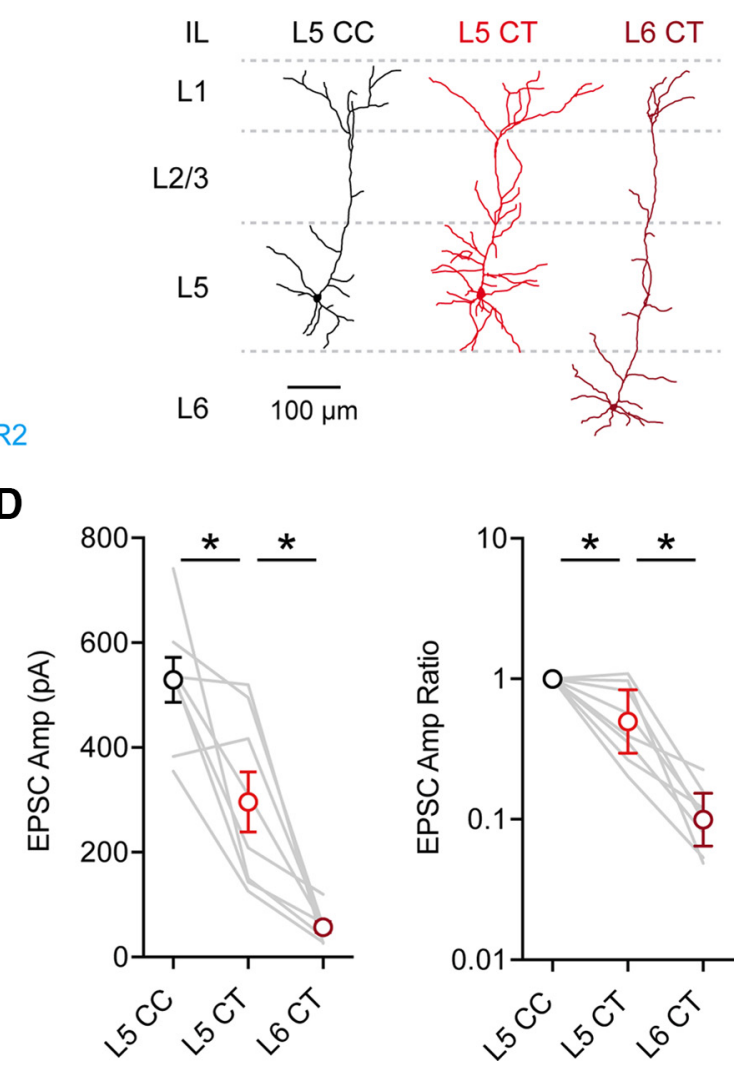

Figure 6. $\quad \mathrm{VHPC}$ inputs are weaker onto $C T$ neurons in both $L 5$ and $L 6 . A, A A V-C h R 2$ injection into the $v H P C$, and coinjection of red

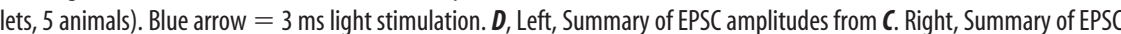
amplitude ratios from $\boldsymbol{C}$. Note the logarithmic axis. ${ }^{*} p<0.05$.

20, 8 and $4 \mathrm{~Hz}$, as these frequencies cover the range where synchronized theta hippocampal-prefrontal oscillations peak in working memory tasks (Adhikari et al., 2010; O'Neill et al., 2013).

We observed that repetitive stimulation of vHPC inputs evoked sustained EPSCs at $-70 \mathrm{mV}$ and IPSCs at $+10 \mathrm{mV}$ at IL L5 CC neurons (Fig. 9A). Over the course of trains, EPSCs showed frequency-dependent facilitation, which was most prominent at $20 \mathrm{~Hz}$, and largely absent at $4 \mathrm{~Hz}$ (Fig. 9B; EPSC $_{2} /$ EPSC $_{1}: 20 \mathrm{~Hz}=1.21 \pm 0.05 ; 8 \mathrm{~Hz}=1.14 \pm 0.07 ; 4 \mathrm{~Hz}$ $=1.01 \pm 0.03 ; n=9$ cells, 3 animals $)$. In contrast, IPSCs tended to show gradual depression over a range of frequencies (Fig. 9B; IPSC 2 /IPSC 1 : $20 \mathrm{~Hz}=1.01 \pm 0.09 ; 8 \mathrm{~Hz}=0.83 \pm$ $0.05 ; 4 \mathrm{~Hz}=0.85 \pm 0.03)$. These diverging dynamics increased the excitation/inhibition (E/I) ratio over the course of trains at a range of frequencies $\left(\mathrm{E} / \mathrm{I}_{1}\right.$ vs $\mathrm{E} / \mathrm{I}_{5}: 20 \mathrm{~Hz}=0.53 \pm 0.08 \mathrm{vs}$ $0.73 \pm 0.08 ; 8 \mathrm{~Hz}=0.49 \pm 0.08$ vs $0.83 \pm 0.14 ; 4 \mathrm{~Hz}=0.51 \pm$ 0.10 vs $0.75 \pm 0.15)$. Finally, we found that vHPC trains evoked pronounced EPSPs and AP firing at IL L5 CC neurons, even with intact inhibition (Fig. 9C). The increase in AP probability was also frequency dependent, and again largest at $20 \mathrm{~Hz}$ (Fig. 9D; Pulse ${ }_{2}$ AP probability: $20 \mathrm{~Hz}=0.93 \pm 0.03$; $8 \mathrm{~Hz}=0.56 \pm 0.11 ; 4 \mathrm{~Hz}=0.25 \pm 0.05 ; n=9$ cells, 3 animals $)$. Together, these results indicate that vHPC inputs are able to robustly activate the $\mathrm{CC}$ network during repetitive activity, even in the presence of feedforward inhibition, with synaptic responses strongly depending on frequency. 
A
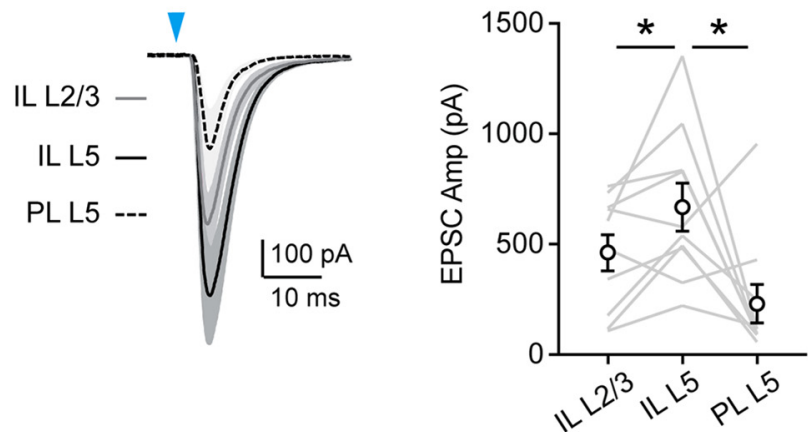

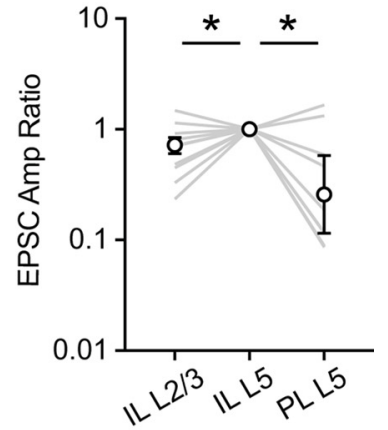

B

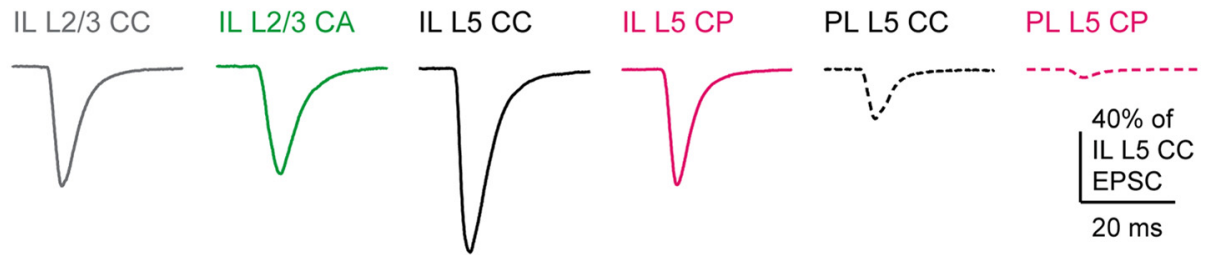

C

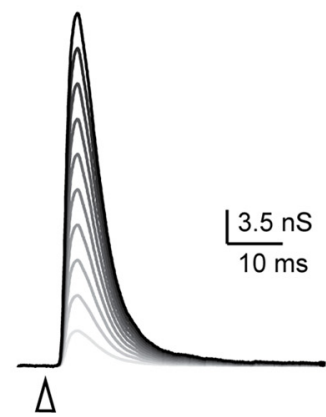

D

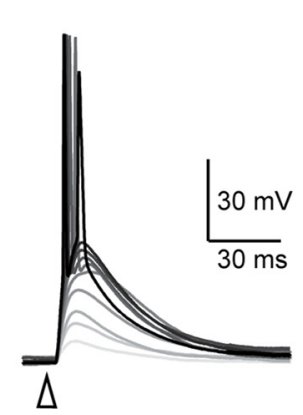

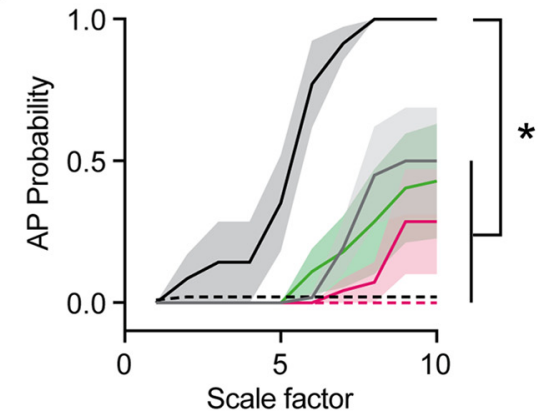

Figure 7. vHPC inputs preferentially activate L5 CC neurons in IL. A, Left, Average vHPC-evoked EPSCs recorded from triplets of CC neurons in IL L2/3, IL L5 and PL L5. Blue arrow $=3$ ms light stimulation. Middle, Summary of EPSC amplitudes. Right, Summary of EPSC amplitude ratios ( $n=10$ triplets, 5 animals). Note the logarithmic axis. $\boldsymbol{B}$, vHPC-evoked EPSCs in the six studied cell types, normalized to responses in IL L5 CC neurons, based on the geometric means of their vHPC-evoked EPSC amplitude ratios from experiments in Figures 3, 4, and 6.C, Left, Synaptic conductances used for dynamic-clamp recordings in ILL5CC neurons, shown across a range of linear scale factors (1-10X), corresponding to conductances of $3.5-35 \mathrm{nS}$. Right, AP firing of an IL L5CC neuron in response to these synaptic conductances at resting membrane potential ( $n=7$ cells, 3 animals). $\boldsymbol{D}$, Summary of AP firing probability of the six cell types shown in $\boldsymbol{B}$, as a function of scale factor ( $n=7$ cells, 3 animals for each cell type). ${ }^{*} p<0.05$.

\section{Discussion}

We have determined the connections made by vHPC inputs onto specific regions and cell types in the mouse PFC. We first showed that vHPC inputs innervate cells in both superficial and deep layers of IL, but only the deep layers of PL. We then determined that this projection is cell-type-specific, contacting both CA and CC neurons in L2/3 of IL, but predominantly CC neurons in L5 of both IL and PL. vHPC inputs also engage local interneurons, leading to feedforward inhibition onto these different projection neurons. Ultimately, these inputs most strongly activate CC neurons in L5 of IL, in either the presence or absence of inhibition. Finally, this influence is frequency dependent, with synaptic facilitation strongly enhancing AP firing during repetitive activity. Together, our findings highlight how vHPC inputs preferentially engage and drive activity of CC neurons in the IL subdivision of the PFC (Fig. 9E).

Our retrograde anatomy showed that hippocampal innervation of PFC derives from vHPC, with minimal input from dHPC. These findings suggest that any influence of dHPC on the PFC is likely to be indirect, through an intermediate brain region, such as the lateral entorhinal cortex or nucleus reuniens (Burwell and Amaral, 1998; Vertes et al., 2007). In contrast, our anterograde anatomy showed that vHPC densely innervates IL, with sparser axons in PL. These findings are consistent with both vHPC and ventral medial PFC being involved in emotional control (Heidbreder and Groenewegen, 2003; Fanselow and Dong, 2010; Euston et al., 2012). PFC subregions also differentially influence fear learning, with IL primarily responsible for extinction (Burgos-Robles et al., 2007; Sotres-Bayon and Quirk, 2010; DoMonte et al., 2015), and PL enabling fear expression (BurgosRobles et al., 2009). The preferential targeting of IL observed in our study could help explain why vHPC inputs have a particularly strong impact on fear extinction (Peters et al., 2010; Sotres-Bayon et al., 2012; Marek et al., 2018). Although it is challenging to selectively target PL and IL with stereotaxic injections to study their subcircuitry in mice, in the future it will be interesting to address whether PL and IL are contacted by distinct populations of hippocampal projection neurons.

Throughout the cortex, superficial and deep layers are thought to compute different input/output functions (Weiler et al., 2008; Harris and Shepherd, 2015). While neurons in L2/3 PL have been shown to respond to vHPC inputs (Little and Carter, 2012), our findings indicate this input is relatively weak. Instead, vHPC inputs preferentially contact pyramidal neurons in $\mathrm{L} 2 / 3$ 
A

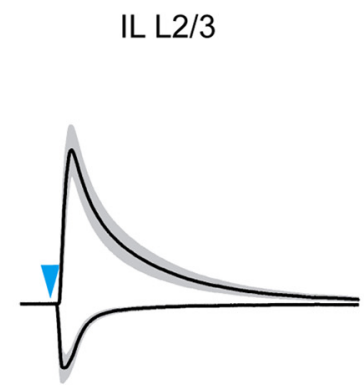

B

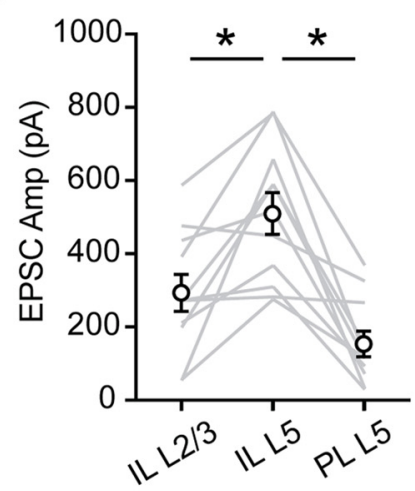

IL L5

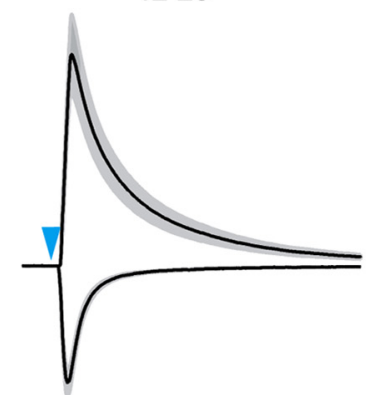

PL L5

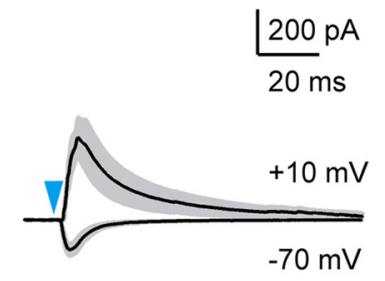

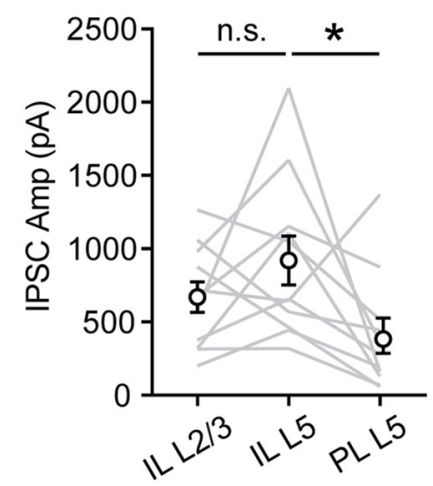

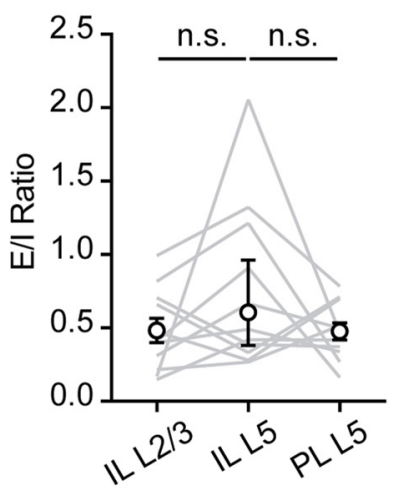

C

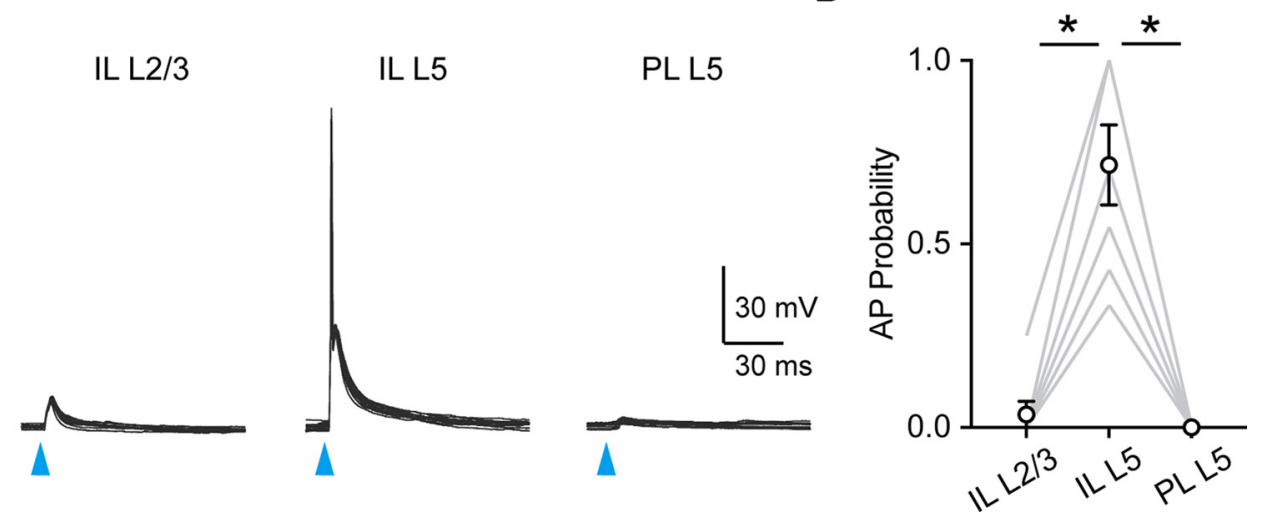

Figure 8. Activation of IL L5 CC neurons persists in the presence of inhibition. A, Average vHPC-evoked EPSCs at $-70 \mathrm{mV}$ and IPSCS at $+10 \mathrm{mV}$, recorded from triplets of IL L2/3 CC neurons, IL L5 CC neurons and PL L5 CC neurons. Blue arrow $=3 \mathrm{~ms}$ light stimulation. B, Left, Summary of EPSC amplitudes. Middle, Summary of IPSC amplitudes. Right, Summary of E/I ratio $(n=11$ triplets, 6 animals). C, vHPC-evoked EPSPs and APs recorded in current-clamp at resting membrane potentials from an example triplet of CC neurons, with 10 traces superimposed for each cell. $\boldsymbol{D}$, Summary of AP firing probability ( $n=7$ triplets, 3 animals). ${ }^{*} p<0.05$.

and L5 of IL, but primarily L5 of PL, with minimal targeting to L6 neurons of either region, even though L6 neurons extend their dendrites into the hippocampal axonal field. Importantly, this bias would likely be missed if responses from L5 were pooled with L6, as the latter receives little input. Notably, this innervation pattern also differs from other long-range afferents to the PFC, whose axons arborize in different layers. For example, callosal inputs innervate multiple layers (Anastasiades et al., 2018), thalamic inputs target superficial layers (Cruikshank et al., 2012; Delevich et al., 2015; Collins et al., 2018), and BLA targets deep IL and superficial PL (Little and Carter, 2013; Cheriyan et al., 2016). These different patterns of connectivity indicate that different long-range inputs activate distinct microcircuits and trigger unique network dynamics across layers of PFC.

Our recordings also highlight how vHPC inputs contact specific populations of projection neurons in the PFC. vHPC inputs are equally strong at L2/3 CC and CA neurons in IL, reminiscent of callosal inputs to these neurons in PL (Little and Carter, 2013). However, this targeting is very different from BLA inputs, which preferentially contact L2/3 CA neurons (Little and Carter, 2013; McGarry and Carter, 2016). By targeting different regions and projection neurons, vHPC and BLA inputs will have distinct influences on the network, contributing to their divergent impact on PFC control of emotion (Sotres-Bayon et al., 2012). vHPC inputs to L5 are strongly biased onto CC over CP neurons in both IL and PL, in marked contrast to callosal inputs to PL (Anastasiades et al., 2018) and BLA inputs to IL (Cheriyan et al., 2016). Importantly, CC neurons also send axons collaterals to other intratelencephalic targets, including the striatum and claustrum (Harris and Shepherd, 2015). Thus, vHPC activation of CC neurons may enable local cortical processing and engage corticoclaustral and cortico-striatal pathways. vHPC inputs have limited 
A

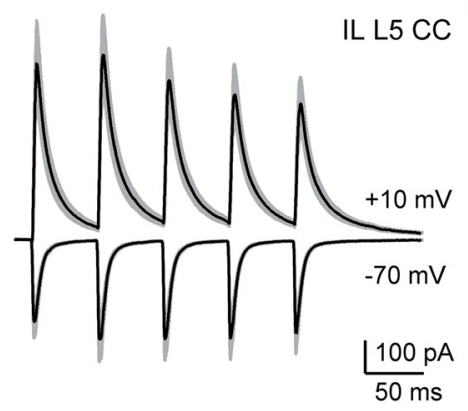

C

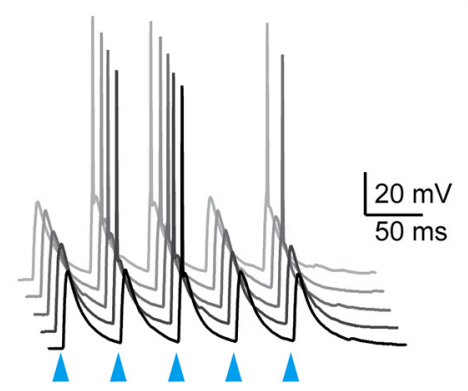

B
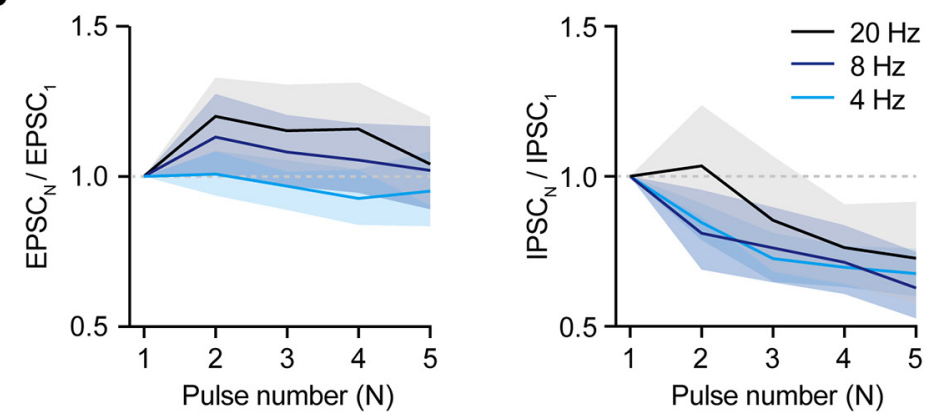

E

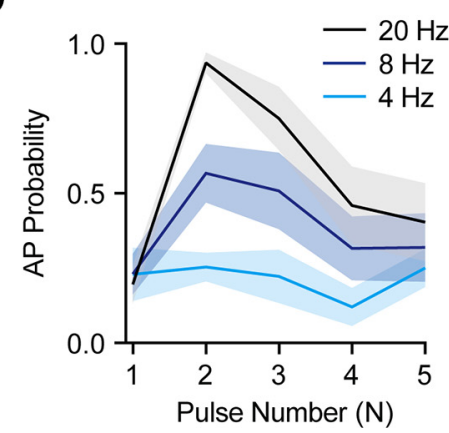

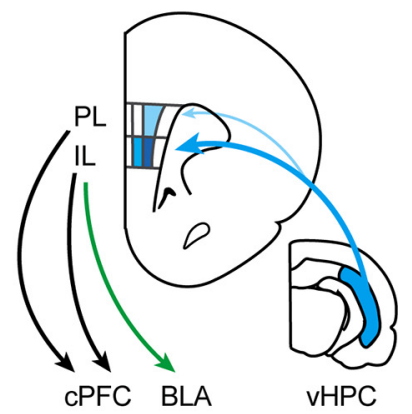

Figure 9. VHPC activation of IL L5 CC neurons depends on input frequency. $A$, Average vHPC-evoked $20 \mathrm{~Hz}$ trains of EPSCs at $-70 \mathrm{mV}$ and IPSCS at $+10 \mathrm{mV}$ at IL L5 CC neurons $(n=9$ cells, 3 animals). $\boldsymbol{B}$, Left, Summary of EPSC PPR (EPSC $/$ EPSC $C_{1}$ ) as a function of pulse number for 20, 8 , and $4 \mathrm{~Hz}$ trains. Right, Similar for IPSC PPR. Gray dashes line: EPSC $_{\mathbb{N}} /$ EPSC $_{1}=1 . C_{\text {, vHPC-evoked } 20 \mathrm{~Hz}}$ trains of EPSPs and APs in an example IL L5 CC neuron, recorded in current-clamp at resting membrane potential, with five staggered traces. Blue arrow: five $3 \mathrm{~ms}$ light pulses at $20 \mathrm{~Hz}$. D, Summary of AP firing probability as a function of pulse number for 20,8 , and $4 \mathrm{~Hz}$ trains ( $n=9$ cells, 3 animals). $E$, Summary schematic of vHPC to PFC projections. vHPC inputs activate CC and CA networks by targeting superficial and deep IL, along with deep PL.

direct access to cortico-thalamic pathway both in L5 and L6, suggesting they must first be processed by the local cortical circuit to influence CT neurons in this circuit (Collins et al., 2018). Stronger inputs onto CC neurons are enhanced by the intrinsic properties of these cells, generating much larger EPSPs and AP firing in this subpopulation (Anastasiades et al., 2018). These comparisons highlight how different long-range inputs can selectively access projection neurons, providing a circuit-level substrate for integrative computations in top-down control (Miller and Cohen, 2001).

Understanding the targeting of vHPC inputs proved critical for assessing how different types of projection neurons are activated. For example, although responses in L2/3 and L5 IL initially appeared similar, this was only because unlabeled L5 neurons contained both highly contacted CC neurons and poorly contacted CP neurons. Comparing synaptic strength in triplets of CC neurons showed a hierarchy of synaptic strength, with IL L5 CC > IL L2/3 CC > PL L5 CC. Although our finding that vHPC inputs target CC neurons in L5 agrees with previous studies (Dembrow et al., 2015), we observed interesting differences between IL and PL. First, the bias onto CC neurons over CP neurons is much larger in PL, with similar results observed for other pyramidal tract neurons, including those that project to the thalamus or the VTA (data not shown). Second, vHPC inputs preferentially target the dendrites of L5 CP neurons, with the strongest targeting observed in IL, consistent with the presence of vHPC inputs to the superficial IL, which allows them to be sampled by these neurons. However, we did not observe any vHPC inputs to the most distal dendrites of either CC or CP neurons, consistent with their relative absence in L1. Although we did not assess dendritic filtering in detail, our approach showed the majority of vHPC inputs contact perisomatic areas of L5 neurons, similar to cPFC inputs (Anastasiades et al., 2018) but in contrast to thalamic inputs (Collins et al., 2018). Overall, our findings indicate important differences in the targeting of IL and PL, which could be missed when pooling recordings from both of these regions.

In many of our experiments, we isolated monosynaptic responses to assess direct input strength at different neurons. However, long-range inputs to the PFC can also strongly engage local GABAergic interneurons to drive feedforward inhibition (Cruikshank et al., 2012; Delevich et al., 2015; McGarry and Carter, 2016; Anastasiades et al., 2018; Collins et al., 2018). Recent studies suggest that vHPC predominantly engage PV-expressing interneurons in the PFC to mediate behavioral effects (Marek et al., 2018). We observed that vHPC inputs evoke robust feedforward inhibition at CC neurons, which is largely balanced with excitation, and again largest in L5 IL. Interestingly, similarly balanced excitation/inhibition ratios across cell types are observed for other long-range inputs to the PFC, including those from the BLA and the contralateral PFC (McGarry and Carter, 2016; Anastasiades et al., 2018). This balance may reflect a universal theme for the regulation of reliable information processing in neocortices (Isaacson and Scanziani, 2011; Yizhar et al., 2011). In the future, it will be important to further assess which interneurons are engaged by vHPC inputs and determine how the balance of excitation and inhibition changes over the course of development and after learning (Sotres-Bayon et al., 2012; Li et al., 2013; Xue et al., 2014).

In the intact brain, hippocampal neurons fire at high frequencies (Siapas et al., 2005), generating streams of inputs to pyramidal neurons and interneurons. Synchronized theta oscillations between hippocampus and the PFC are particularly important for working memory (Siapas et al., 2005; O’Neill et al., 2013; 
Sigurdsson and Duvarci, 2015). Our recordings demonstrate that vHPC-evoked EPSCs are facilitating, and this short-term plasticity grows with frequency, with greatest magnitude at $20 \mathrm{~Hz}$, which is often used for in vivo optogenetics (Do-Monte et al., 2017; Roy et al., 2017). In contrast, feedforward IPSCs are depressing, suggesting that inhibitory responses may be mediated by fast-spiking interneurons, which often receive depressing inputs, and make depressing synapses onto pyramidal neurons (Reyes et al., 1998; McGarry and Carter, 2016). These dynamics resulted in an elevated $\mathrm{E} / \mathrm{I}$ ratio over the course of stimulus trains, allowing PFC neurons to become further engaged, supporting the dominance of theta frequency in synchronizing vHPC-PFC activity during working memory tasks. Together, these findings suggest that correlated bouts of hippocampal activity are likely to strongly activate the local microcircuit.

In summary, our study reveals the region- and cell-typespecific synaptic organization of hippocampal inputs to the PFC. The hippocampal-prefrontal pathway is important for both cognition and emotion (Euston et al., 2012), and our findings suggest that direct activation of specific populations of CC neurons by vHPC inputs plays an important role. Normal function of this pathway supports working memory and emotional learning (Siapas et al., 2005; Sotres-Bayon et al., 2012; O'Neill et al., 2013; Spellman et al., 2015). Aberrant activity contributes to anxietyrelated behaviors and cognitive deficits (Adhikari et al., 2010; Sigurdsson et al., 2010; Godsil et al., 2013; Sigurdsson and Duvarci, 2015; Padilla-Coreano et al., 2016), which may reflect the lack or disruption of regulated hippocampal drive that maintains normal activity in the PFC. Our results suggest that IL is particularly important in these interactions, motivating future studies to focus on this area. Moreover, our experiments highlight the importance of considering how inputs contact different regions, layers, and cell types in the PFC, and cortical circuits in general.

\section{References}

Adhikari A, Topiwala MA, Gordon JA (2010) Synchronized activity between the ventral hippocampus and the medial prefrontal cortex during anxiety. Neuron 65:257-269. CrossRef Medline

Anastasiades PG, Marlin JJ, Carter AG (2018) Cell-type specificity of callosally evoked excitation and feedforward inhibition in the prefrontal cortex. Cell Rep 22:679-692. CrossRef Medline

Avesar D, Gulledge AT (2012) Selective serotonergic excitation of callosal projection neurons. Front Neural Circuits 6:12. CrossRef Medline

Burgos-Robles A, Vidal-Gonzalez I, Santini E, Quirk GJ (2007) Consolidation of fear extinction requires NMDA receptor-dependent bursting in the ventromedial prefrontal cortex. Neuron 53:871-880. CrossRef Medline

Burgos-Robles A, Vidal-Gonzalez I, Quirk GJ (2009) Sustained conditioned responses in prelimbic prefrontal neurons are correlated with fear expression and extinction failure. J Neurosci 29:8474-8482. CrossRef Medline

Burwell RD, Amaral DG (1998) Cortical afferents of the perirhinal, postrhinal, and entorhinal cortices of the rat. J Comp Neurol 398:179-205. CrossRef Medline

Carter AG, Regehr WG (2002) Quantal events shape cerebellar interneuron firing. Nat Neurosci 5:1309-1318. CrossRef Medline

Chalifoux JR, Carter AG (2010) GABAB receptors modulate NMDA receptor calcium signals in dendritic spines. Neuron 66:101-113. CrossRef Medline

Cheriyan J, Kaushik MK, Ferreira AN, Sheets PL (2016) Specific targeting of the basolateral amygdala to projectionally defined pyramidal neurons in prelimbic and infralimbic cortex. eNeuro 3:ENEURO.0002-16.2016. CrossRef Medline

Collins DP, Anastasiades PG, Marlin JJ, Carter AG (2018) Reciprocal circuits linking the prefrontal cortex with dorsal and ventral thalamic nuclei. Neuron 98:366-379.e4. CrossRef Medline

Cruikshank SJ, Ahmed OJ, Stevens TR, Patrick SL, Gonzalez AN, Elmaleh M,
Connors BW (2012) Thalamic control of layer 1 circuits in prefrontal cortex. J Neurosci 32:17813-17823. CrossRef Medline

Delevich K, Tucciarone J, Huang ZJ, Li B (2015) The mediodorsal thalamus drives feedforward inhibition in the anterior cingulate cortex via parvalbumin interneurons. J Neurosci 35:5743-5753. CrossRef Medline

Dembrow NC, Chitwood RA, Johnston D (2010) Projection-specific neuromodulation of medial prefrontal cortex neurons. J Neurosci 30:1692216937. CrossRef Medline

Dembrow NC, Zemelman BV, Johnston D (2015) Temporal dynamics of L5 dendrites in medial prefrontal cortex regulate integration versus coincidence detection of afferent inputs. J Neurosci 35:4501-4514. CrossRef Medline

Do-Monte FH, Manzano-Nieves G, Quiñones-Laracuente K, Ramos-Medina L, Quirk GJ (2015) Revisiting the role of infralimbic cortex in fear extinction with optogenetics. J Neurosci 35:3607-3615. CrossRef Medline

Do-Monte FH, Minier-Toribio A, Quinones-Laracuente K, Medina-Colón EM, Quirk GJ (2017) Thalamic regulation of sucrose seeking during unexpected reward omission. Neuron 94:388-400.e4. CrossRef Medline

Euston DR, Gruber AJ, McNaughton BL (2012) The role of medial prefrontal cortex in memory and decision making. Neuron 76:1057-1070. CrossRef Medline

Fanselow MS, Dong HW (2010) Are the dorsal and ventral hippocampus functionally distinct structures? Neuron 65:7-19. CrossRef Medline

Gabbott PL, Warner TA, Jays PR, Salway P, Busby SJ (2005) Prefrontal cortex in the rat: projections to subcortical autonomic, motor, and limbic centers. J Comp Neurol 492:145-177. CrossRef Medline

Godsil BP, Kiss JP, Spedding M, Jay TM (2013) The hippocampalprefrontal pathway: the weak link in psychiatric disorders? Eur Neuropsychopharmacol 23:1165-1181. CrossRef Medline

Harris KD, Shepherd GM (2015) The neocortical circuit: themes and variations. Nat Neurosci 18:170-181. CrossRef Medline

Hattox AM, Nelson SB (2007) Layer V neurons in mouse cortex projecting to different targets have distinct physiological properties. J Neurophysiol 98:3330-3340. CrossRef Medline

Heidbreder CA, Groenewegen HJ (2003) The medial prefrontal cortex in the rat: evidence for a dorso-ventral distinction based upon functional and anatomical characteristics. Neurosci Biobehav Rev 27:555-579. CrossRef Medline

Hoover WB, Vertes RP (2007) Anatomical analysis of afferent projections to the medial prefrontal cortex in the rat. Brain Struct Funct 212:149-179. CrossRef Medline

Isaacson JS, Scanziani M (2011) How inhibition shapes cortical activity. Neuron 72:231-243. CrossRef Medline

Kim WB, Cho JH (2017) Synaptic targeting of double-projecting ventral CA1 hippocampal neurons to the medial prefrontal cortex and basal amygdala. J Neurosci 37:4868-4882. CrossRef Medline

Kitamura T, Ogawa SK, Roy DS, Okuyama T, Morrissey MD, Smith LM, Redondo RL, Tonegawa S (2017) Engrams and circuits crucial for systems consolidation of a memory. Science 356:73-78. CrossRef Medline

Li H, Penzo MA, Taniguchi H, Kopec CD, Huang ZJ, Li B (2013) Experience-dependent modification of a central amygdala fear circuit. Nat Neurosci 16:332-339. CrossRef Medline

Little JP, Carter AG (2012) Subcellular synaptic connectivity of layer 2 pyramidal neurons in the medial prefrontal cortex. J Neurosci 32:1280812819. CrossRef Medline

Little JP, Carter AG (2013) Synaptic mechanisms underlying strong reciprocal connectivity between the medial prefrontal cortex and basolateral amygdala. J Neurosci 33:15333-15342. CrossRef Medline

Marek R, Jin J, Goode TD, Giustino TF, Wang Q, Acca GM, Holehonnur R, Ploski JE, Fitzgerald PJ, Lynagh T, Lynch JW, Maren S, Sah P (2018) Hippocampus-driven feed-forward inhibition of the prefrontal cortex mediates relapse of extinguished fear. Nat Neurosci 21:384-392. CrossRef Medline

McGarry LM, Carter AG (2016) Inhibitory gating of basolateral amygdala inputs to the prefrontal cortex. J Neurosci 36:9391-9406. CrossRef Medline

Miller EK, Cohen JD (2001) An integrative theory of prefrontal cortex function. Annu Rev Neurosci 24:167-202. CrossRef Medline

Morishima M, Morita K, Kubota Y, Kawaguchi Y (2011) Highly differentiated projection-specific cortical subnetworks. J Neurosci 31:1038010391. CrossRef Medline

O'Neill PK, Gordon JA, Sigurdsson T (2013) Theta oscillations in the me- 
dial prefrontal cortex are modulated by spatial working memory and synchronize with the hippocampus through its ventral subregion. J Neurosci 33:14211-14224. CrossRef Medline

Otsuka T, Kawaguchi Y (2011) Cell diversity and connection specificity between callosal projection neurons in the frontal cortex. J Neurosci 31: 3862-3870. CrossRef Medline

Padilla-Coreano N, Bolkan SS, Pierce GM, Blackman DR, Hardin WD, Garcia-Garcia AL, Spellman TJ, Gordon JA (2016) Direct ventral hippocampal-prefrontal input is required for anxiety-related neural activity and behavior. Neuron 89:857-866. CrossRef Medline

Peters J, Dieppa-Perea LM, Melendez LM, Quirk GJ (2010) Induction of fear extinction with hippocampal-infralimbic BDNF. Science 328:12881290. CrossRef Medline

Petreanu L, Mao T, Sternson SM, Svoboda K (2009) The subcellular organization of neocortical excitatory connections. Nature 457:1142-1145. CrossRef Medline

Pouille F, Scanziani M (2001) Enforcement of temporal fidelity in pyramidal cells by somatic feed-forward inhibition. Science 293:1159-1163. CrossRef Medline

Reyes A, Lujan R, Rozov A, Burnashev N, Somogyi P, Sakmann B (1998) Target-cell-specific facilitation and depression in neocortical circuits. Nat Neurosci 1:279-285. CrossRef Medline

Roy DS, Kitamura T, Okuyama T, Ogawa SK, Sun C, Obata Y, Yoshiki A, Tonegawa S (2017) Distinct neural circuits for the formation and retrieval of episodic memories. Cell 170:1000-1012.e19. CrossRef Medline

Sheets PL, Suter BA, Kiritani T, Chan CS, Surmeier DJ, Shepherd GM (2011) Corticospinal-specific HCN expression in mouse motor cortex: $I_{\mathrm{h}}$ dependent synaptic integration as a candidate microcircuit mechanism involved in motor control. J Neurophysiol 106:2216-2231. CrossRef Medline

Siapas AG, Lubenov EV, Wilson MA (2005) Prefrontal phase locking to hippocampal theta oscillations. Neuron 46:141-151. CrossRef Medline

Sigurdsson T, Duvarci S (2015) Hippocampal-prefrontal interactions in cognition, behavior and psychiatric disease. Front Syst Neurosci 9:190. CrossRef Medline
Sigurdsson T, Stark KL, Karayiorgou M, Gogos JA, Gordon JA (2010) Impaired hippocampal-prefrontal synchrony in a genetic mouse model of schizophrenia. Nature 464:763-767. CrossRef Medline

Sotres-Bayon F, Quirk GJ (2010) Prefrontal control of fear: more than just extinction. Curr Opin Neurobiol 20:231-235. CrossRef Medline

Sotres-Bayon F, Sierra-Mercado D, Pardilla-Delgado E, Quirk GJ (2012) Gating of fear in prelimbic cortex by hippocampal and amygdala inputs. Neuron 76:804-812. CrossRef Medline

Spellman T, Rigotti M, Ahmari SE, Fusi S, Gogos JA, Gordon JA (2015) Hippocampal-prefrontal input supports spatial encoding in working memory. Nature 522:309-314. CrossRef Medline

Tierney PL, Dégenètais E, Thierry AM, Glowinski J, Gioanni Y (2004) Influence of the hippocampus on interneurons of the rat prefrontal cortex. Eur J Neurosci 20:514-524. CrossRef Medline

Tremblay R, Lee S, Rudy B (2016) GABAergic interneurons in the neocortex: from cellular properties to circuits. Neuron 91:260-292. CrossRef Medline

Vertes RP, Hoover WB, Szigeti-Buck K, Leranth C (2007) Nucleus reuniens of the midline thalamus: link between the medial prefrontal cortex and the hippocampus. Brain Res Bull 71:601-609. CrossRef Medline

Weiler N, Wood L, Yu J, Solla SA, Shepherd GM (2008) Top-down laminar organization of the excitatory network in motor cortex. Nat Neurosci 11:360-366. CrossRef Medline

Xue M, Atallah BV, Scanziani M (2014) Equalizing excitation-inhibition ratios across visual cortical neurons. Nature 511:596-600. CrossRef Medline

Yizhar O, Fenno LE, Prigge M, Schneider F, Davidson TJ, O'Shea DJ, Sohal VS, Goshen I, Finkelstein J, Paz JT, Stehfest K, Fudim R, Ramakrishnan C, Huguenard JR, Hegemann P, Deisseroth K (2011) Neocortical excitation/inhibition balance in information processing and social dysfunction. Nature 477:171-178. CrossRef Medline

Zucker RS, Regehr WG (2002) Short-term synaptic plasticity. Annu Rev Physiol 64:355-405. CrossRef Medline 